\title{
Sub-pixel image registration with a maximum likelihood estimator
}

\section{Application to the first adaptive optics observations of Arp 220 in the $L^{\prime}$ band ${ }^{\star}$}

\author{
D. Gratadour ${ }^{1,2}$, L. M. Mugnier ${ }^{1}$, and D. Rouan ${ }^{2}$
}

\author{
1 DOTA - ONERA, Av. de la division Leclerc, Chatillon, France \\ 2 LESIA, Observatoire de Paris, 5 place Jules Janssen, 92195, Meudon, France \\ e-mail: damien.gratadour@obspm.fr
}

Received 15 October 2004 / Accepted 26 July 2005

\section{ABSTRACT}

We present a new method based on a maximum likelihood (ML) estimation of the sub-pixel shift between images of a given object observed with a single instrument. We first study the case of two noisy images and give the ML approach of the registration problem. By means of simulations, we show the gain obtained with this ML solution compared to a classical registration method with an academic noise model (stationary white Gaussian), and then demonstrate the relevance of this ML estimation with a more realistic noise model. We then address the problem of a sequence of low signal frames of the same object. We develop a joint ML approach in which we simultaneously estimate the reference (i.e. the noiseless) image and the shift parameters. The registration accuracy is increased at low photon levels as the number of frames grows, reaching the sub-pixel domain at very low SNR (about 1), when considering 100 frames. When applied to experimental data (thermal IR images of a faint galaxy), both ML methods show their efficiency to recover the resolution in averaged frames and totally outperform the classical cross-correlation.

Key words. techniques: image processing - instrumentation: adaptive optics - galaxies: individual: Arp 220

\section{Introduction}

Image registration at the level of a pixel or less is a common problem in many domains, and the number of developed methods makes it a full-fledged research field (see Brown 1992; and Zitová \& Flusser 2003). Nevertheless, there is no universal solution as the method to use strongly depends on the nature of the transformation between the images to be registered. Registration for medical imaging is probably one of the most diversified fields, with many different types of problems (multi-modal, 3D or stereoscopic imaging for instance), for which different methods have been developed in each case (see Maurer \& Fitzpatrick 1993; and Maintz \& Viergever 1998, for a review). In this paper we focus on the problem of shifted noisy Nyquist-sampled images of the same object observed with the same instrument. Hence, following the general classification (Zitová \& Flusser 2003; Roche et al. 2000) of registration methods, we propose a new area-based (or intensity-based) technique for image registration. The most popular method used in this case is the cross-correlation (CC) between the images (see e.g. Brown 1992; and Zitová \& Flusser 2003, and references therein for a review). If the sub-pixel accuracy is required, interpolation of the $\mathrm{CC}$ function around its

^ Based on observations collected at the ESO/Paranal YEPUN telescope, Proposal 70.B-0307(A). maximum is needed. Many interpolation methods have been tested which have different robustness and accuracy depending on the noise nature and intensity (see Roche et al. 2000; Zitová \& Flusser 2003). For instance, in the case of undersampled frames, Carfantan \& Rougé (2001) have studied different methods to find an unbiased estimation of the sub-pixel shift between two images, and conclude that only the interpolation of the CC function by a cardinal sine does not give a biased estimation. A similar approach is to compute the CC of images after oversampling them (with a cardinal sine or a cubic interpolation for instance). The accuracy of the method depends on the interpolation function. The latter approach is the most used method in astronomy when the images are at least Nyquist-sampled (see e.g. the registration method included in the IDL astro-Lib package from NASA). A classical method also used for astronomical images is the Drizzle algorithm (Fruchter \& Hook 2002), developed for under-sampled HST images, which corrects for misregistration as well as for rotation and distortions. The correction for translation is also based on the CC of the interpolated images and thus suffers from the same limitations: explicit re-sampling and no precise noise model taken into account.

Some authors have proposed registration as a Maximum Likelihood (hereafter ML) problem (Mort \& Srinath 1988; Costa et al. 1993), and Roche et al. (2000) have developed a 


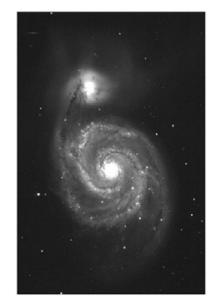

OBJECT [0]

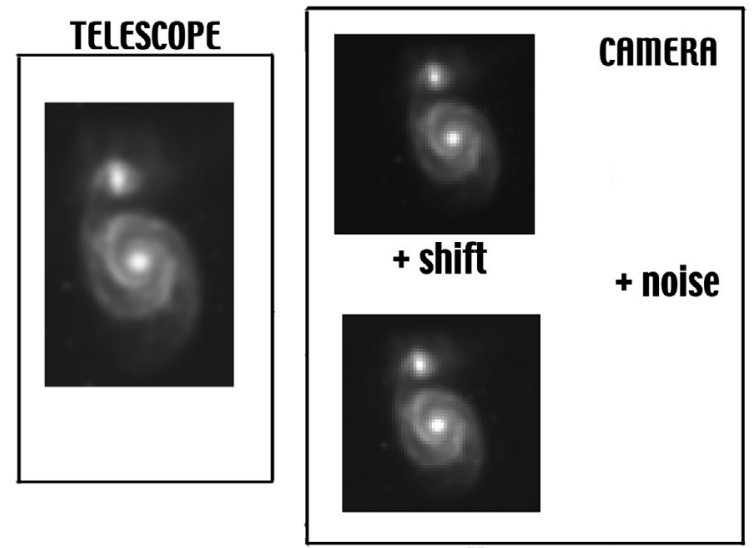

sampling
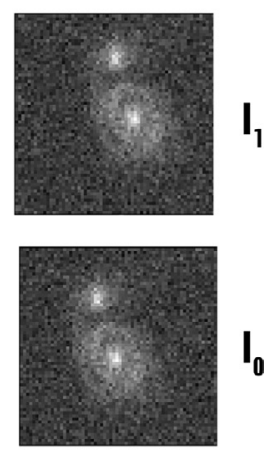

Fig. 1. Scheme describing the formation of an image. The sampling is assumed to verify the Nyquist-Shannon theorem.

general approach for registration problems using a ML formalism. The particular case of two images obtained with the same instrument is not studied because the main issue of their paper is the registration of images acquired under different observing conditions. Closer to our study, in the case of a sequence of translated Poisson limited Nyquist sampled images of the same object, a joint ML method has been developed to restore the reference image and the shift parameters of the sequence simultaneously (Guillaume et al. 1998).

The registration problem also occurs when collecting several short exposure images under weak turbulent conditions. Indeed, under such conditions, the effect of turbulence is mainly a tip-tilt on the wavefront and thus a translation of the images. This problem is addressed in the seminal paper of Snyder \& Schulz (1990), in which they describe the EM algorithm they used to restore a high resolution image from a sequence of translated short exposures. This work and the work of Guillaume et al. (1998) are dedicated to extremely low photon levels (a few photons per image) whereas we consider images with a few photons per pixel corrupted by background and detector noise. With the greater photon level we consider, we aim at sub-pixel accuracy even when the object flux is comparable to the noise variance (detector+background noise). Moreover, when the problem allows such an approach, conjugate gradient algorithms are usually recognised to be faster than EM.

Some authors have also considered registration as part of a more global restoration problem, and propose ML approaches, depending on the images properties and the nature of the transformation, and including the registration problem. Recently, Girémus \& Carfantan (2003) have developed a multiframe deconvolution algorithm, for which sub-pixel accuracy is required, and propose a joint ML estimator of the images and the shift parameters, but only in the case of stationary white Gaussian noise. They note that in the case of low SNR, the ML method for the estimation of the shift parameters gives more accurate results than classical empirical estimators. However, if we consider a PSF almost constant in time, as in the case of adaptive optics (hereafter AO) observations, no gain is expected by jointly registering and restoring as demonstrated by Girémus \& Carfantan (2003). It is obviously less costly to register first all the frames and then deconvolved the average (long exposure) image.

This paper is organized as follows. We first describe our image model and introduce the ML formalism in the simple case of registering two images. We test our method with simulated images, in the case of stationary white Gaussian noise as well as with a more realistic mixture of Poissonian and stationary Gaussian noise. In Sect. 3, we address the problem of registering a sequence of images and develop a joint maximum likelihood approach to estimate simultaneously the shift parameters and the reference image. We then demonstrate the efficiency of this method with simulated images. In Sect. 4, we use various methods to register very noisy images of a distant galaxy acquired with an AO system in the thermal infrared and we compare the results. We conclude in the last section.

\section{Description of the method}

\subsection{Data model}

In the following, we use a one dimensional development for clarity. This section aims at presenting the context from which we naturally introduce the ML approach. The simplest statistical model of the data is obtained when one of the two images is considered to be noiseless, and the other one is a shifted and noisy version of the former. An illustration of this scheme is presented in Fig. 1.

We consider an object $O(x)$ seen through an instrument of known PSF $H(x)$. The latter includes the effect of the propagating medium, the telescope and the detector. Assuming no detection noise, a reference image is then $R(k)=$ $[R(x)]_{\amalg}(k)=[O(x) * H(x)]_{\amalg}(k)$, where $\amalg$ is the sampling operator, $[R(x)]_{\amalg}(k)$ is the $k$ th sample of the function $R(x)$ and $*$ the convolution operator. We will assume that the sampling process respects the Shannon-Nyquist criterion, meaning that the highest spatial frequency in the image is at most half the sampling frequency. This implies that a continuous version of the image can be reconstructed via the Shannon reconstruction theorem. A shifted and noisy version of the reference 
(acquired with a finite exposure time after jittering the telescope), is then written:

$$
\begin{aligned}
I_{1}(k) & =\left[R(x) * \delta\left(x-x_{1}\right)\right]_{\amalg}(k)+N_{1}(k) \\
& =\left[R\left(x-x_{1}\right) \times \amalg(x)\right](k)+N_{1}(k)
\end{aligned}
$$

where $x_{1}$ is the shift parameter and $N_{1}$ an additive noise.

\subsection{Gaussian noise - formulation of the pairwise ML approach}

In this section, we present the standard ML formulation of the registration problem in the image formation scheme described above. We will name this approach pairwise ML, as we process pairs of images, to distinguish this method from the one developed in Sect. 3. If we assume a Gaussian additive noise, the likelihood of observing an image $I_{1}(k)$ for the reference (noiseless image) $R(x)$ and for the hypothesis $x_{1}$ is given by:

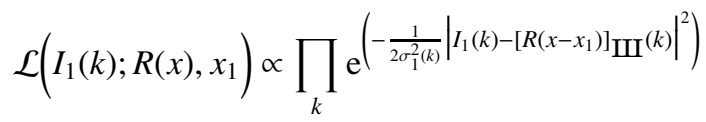

where $\sigma_{1}^{2}$ is a map of the noise variance (see for instance Sect. 3 in Van Trees 1968). The negative log-likelihood is then:

$\mathcal{J}\left(x_{1}\right)=\sum_{k} \frac{1}{2 \sigma_{1}^{2}(k)}\left|I_{1}(k)-\left[R\left(x-x_{1}\right)\right]_{\amalg}(k)\right|^{2}$

and the ML estimate $\hat{x}_{1_{\mathrm{ML}}}$ of the shift between the two images is given by minimizing numerically $\mathcal{J}\left(x_{1}\right)$ by, e.g., a conjugate gradient method.

\subsubsection{Stationary white Gaussian noise: the cross-correlation}

If the noise is additionally stationary, which is for instance approximatively the case for background dominated noise if the background is uniform (thermal IR observations for instance) or for low photon level images dominated by detector noise, the pairwise ML solution of the registration problem is known to be the maximum of the cross-correlation between the two images, assuming their periodicity (see Appendix A).

The formulation of the registration in a ML framework suggests an implementation of the solution that can be arbitrarily sub-pixel without any explicit resampling of the images, and which turns out to be more precise than readily available (IDL astro library, for instance) code, as shown in next section.

\subsubsection{Generalization to non-stationary Gaussian noise}

We now consider a more realistic noise model describing astronomical observations in the infrared. In this framework, the global noise on the images can be decomposed in two components: a Poisson distributed noise due to the detection process (object + background) and a Gaussian distributed noise due to the CCD electronics. This case has been previously studied in Snyder et al. (1995), where a sophisticated noise model is considered. In their paper, the distribution of the mixture of Gaussian and Poissonian noises is either approximated by a Poissonian distribution or computed numerically using the saddle point method. The performance of the image restoration ML criteria deduced from this two distributions are very close which demonstrates the robustness of the Poissonian approximation in their study (image reconstruction using one image of the object).

In our study, we use the alternative approach of approximating our mixed noise as non-stationary white Gaussian. Actually, in the case of astronomical infrared imaging, the level of the IR background is comparable to the photon flux of the observed object and is typically over a thousand photons per pixel. We are thus working at high background level and the IR background noise distribution is well approximated by a Gaussian. After background subtraction the noise variance is doubled, and the photon level on the object is of the order of a few photons per pixel (more than the variance of the detector noise, i.e. typically more than ten for nowaday's detectors).

In the case described above, the global noise variance can be estimated directly from the images as in Mugnier et al. (2004). The variance of the detector component is estimated beforehand, on a so-called dark image, and the photon noise variance is estimated as:

$\sigma_{\mathrm{ph}}^{2}(k)=\max [I(k), 0]$.

We obtain the pairwise ML solution of the shift by minimizing numerically the criterion of Eq. (1) (and the periodic assumption is no longer needed). With a map of the noise variance, estimated as just described, the ML estimation of the shifts is easier than in the study of Snyder \& Schulz (1990) in which the noise variance and the shifts are estimated simultaneously.

\subsubsection{Generalization to an unknown reference}

In practice, the noiseless reference $R$ is not available. Instead, let us now consider a noisy frame $I_{0}$ :

$I_{0}(k)=[R(x)]_{\amalg}+N_{0}(k)$

assumed to be a noisy version of the reference. A shifted noisy image $I_{1}$ can be written:

$$
\begin{aligned}
I_{1}(k) & =\left[R(x) * \delta\left(x-x_{1}\right)\right]_{\amalg}+N_{1}(k) \\
& =\left[I_{0}(x) * \delta\left(x-x_{1}\right)\right]_{\amalg}+N(k)
\end{aligned}
$$

where: $N(k)=N_{1}(k)-\left[N_{0}(x) * \delta\left(x-x_{1}\right)\right]_{\amalg}$. If we assume $N_{0}$ as a Gaussian distribution of variance $\sigma_{0}^{2}(x)$ and $N_{1}$ the noise of the same detector, then we have: $\sigma_{0}^{2}(x) * \delta\left(x-x_{1}\right)=\sigma_{1}^{2}(x)$.

As the noise variance of $I_{1}$ is estimated on the image, and that $\sigma_{0}^{2}(x)$ is deduced from this estimation, the noise variance is estimated before the minimization of $\mathcal{J}\left(x_{1}\right)$ and thus no more depends on the shift parameters. The neg-log-likelihood to be minimized has so the same expression as in Eq. (1) changing $R(x)$ into $I_{0}(x)$ and $\sigma_{1}^{2}(k)$ into $\sigma^{2}(k)=\sigma_{1}^{2}(k)+$ $\left[\sigma_{0}^{2}(x) * \delta\left(x-x_{1}\right)\right]_{\mathrm{WI}}=2 \sigma_{1}^{2}(k)$ :

$\mathcal{J}\left(x_{1}\right)=\sum_{k} \frac{1}{4 \sigma_{1}^{2}(k)}\left|I_{1}(k)-\left[I_{0}\left(x-x_{1}\right)\right]_{\amalg}\right|^{2}$. 

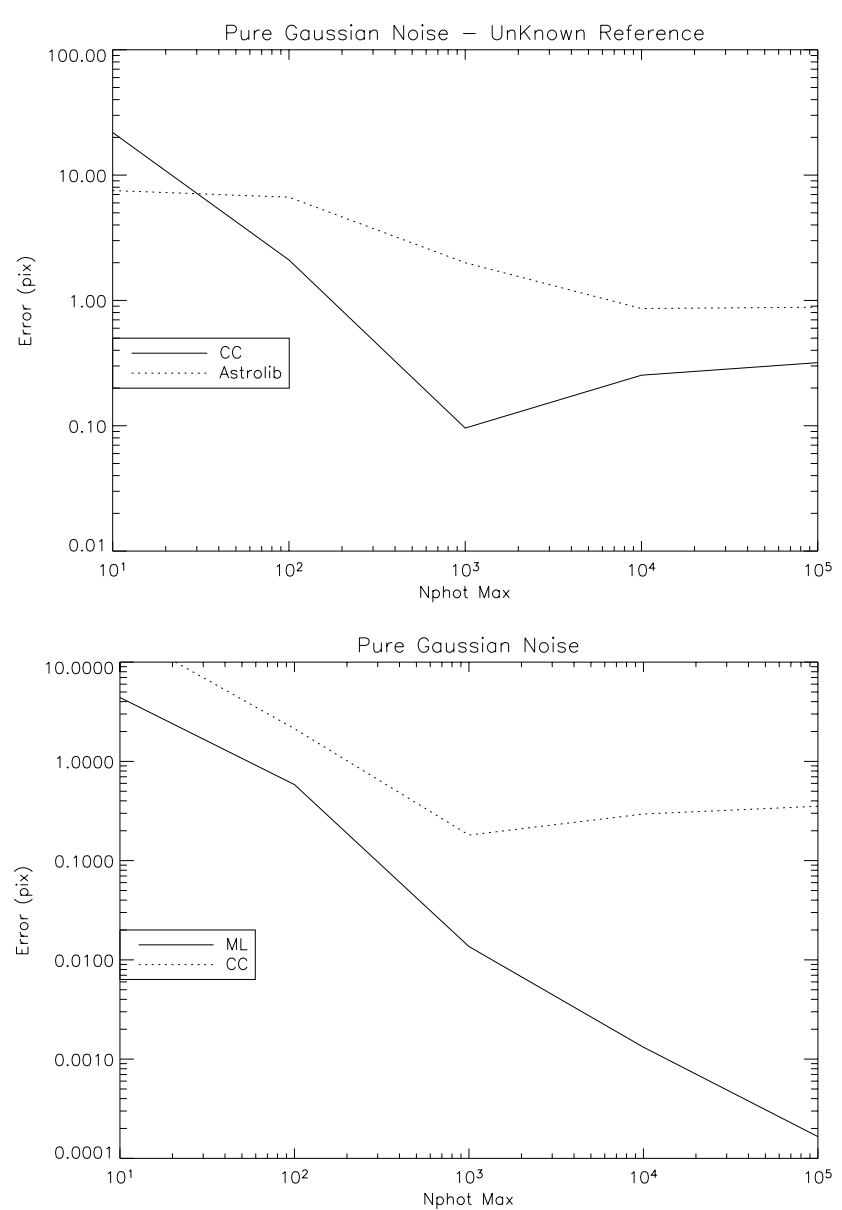

Fig. 2. Top: performance of the IDL astro-Lib registration method in the case of purely Gaussian additive noise compared to the $\mathrm{CC}$ we implemented. Bottom: respective error of each method in the same conditions, solid line, the pairwise ML method, dotted line, the classical cross-correlation method we implemented. The variance of the Gaussian noise is kept a constant at 100 , while the number of photons max per pixel in the initial images ranged from 10 to $10^{5}$.

We see that even if the 2 images are noisy, one of them can still serve as a reference for the other and that the ML solution just derived is still valid (see Fig. 3).

\subsection{Implementation and validation with simulated images}

In order to compare our method to commonly used ones in this kind of registration problems, we implemented (in IDL) the minimization of the criterion of Eq. (1). We compared it to an easy-to-implement cross-correlation method which consists of a discrete computation of the CC function followed by a fit of the latter around its maximum by a continuous function. We chose a Gaussian over a second order polynomial to fit the CC function, in a $2 \times 2$ pixel box around its maximum. When compared to a classical image interpolation-based method (the one included in the IDL astro-library for instance) this CC method is obviously less time consuming and has better performance (see Fig. 2 top). This CC method thus seems a robust and fast member of the classical CC methods family and so is a relevant test competitor to compare to our pairwise ML method.
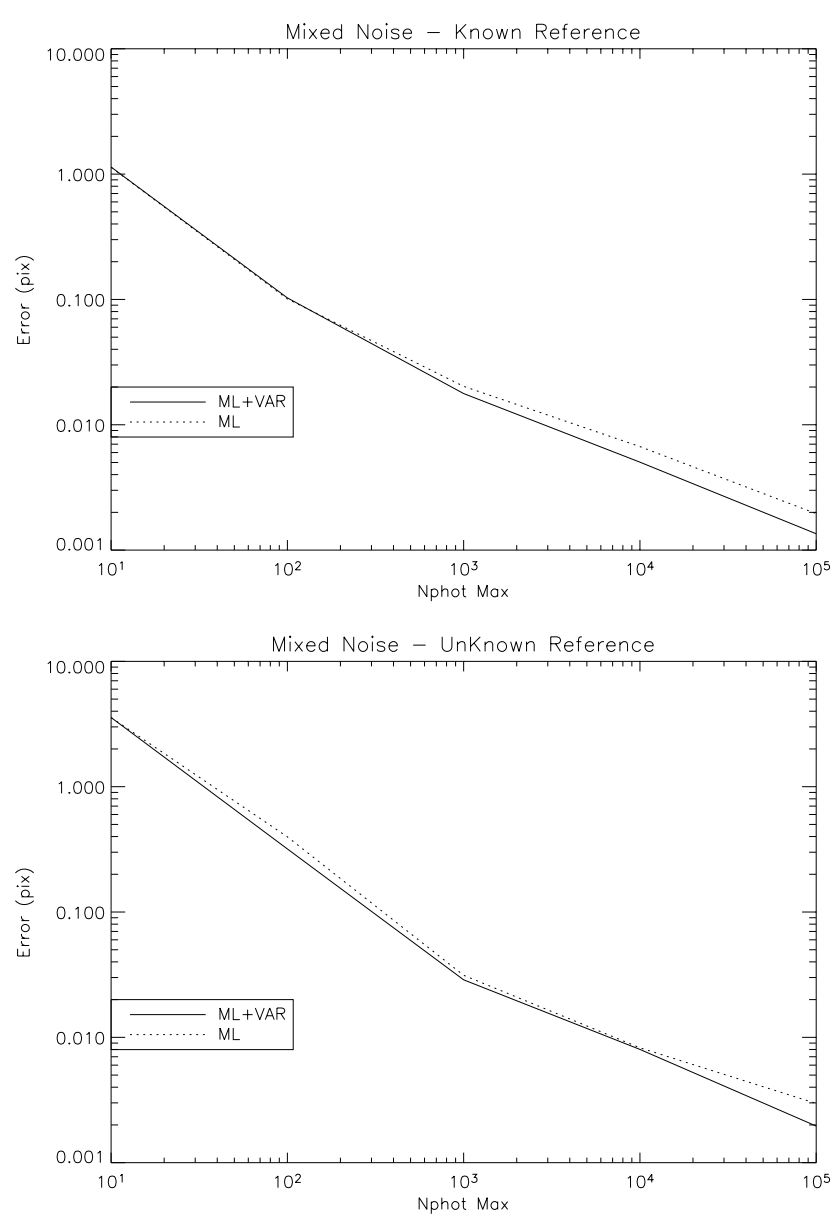

Fig. 3. Performance of the pairwise ML method in the case of mixed (stationary Gaussian + Poissonian) noise. The variance of the Gaussian noise is kept constant to 100 , while the max number of photons per pixel in each image ranges from 10 to $10^{5}$. Solid line: the ML method considering the noise variance map, dotted line: the ML method considering a constant variance. Top, the academic case of a known reference and bottom, the realistic case of an unknown one.

If the images are Nyquist sampled, the Fourier transforms (FT) of the sampled images coincide with the FT of the continuous images within $\left[-\frac{v_{\mathrm{s}}}{2}, \frac{v_{\mathrm{s}}}{2}\right]$ where $v_{\mathrm{s}}$ is the sampling frequency. Therefore, we are able to reconstruct a sub-pixel shifted version of any Nyquist sampled image, by multiplying the phase of the FT of this image by a tilt whose slope is a non integer number. In practice, we used the Fast FT (FFT) algorithm to make the computation faster. This algorithm computes a Discrete FT (DFT) which represents a small approximation. In order to remove noise and to reduce the effect of this approximation at high frequencies, we have found it useful to low-pass filter the noisy reference before applying the above-mentioned tilt to its phase. The cut-off is chosen to equal the diffraction cut-off frequency of the telescope, so that only noise is removed from this image. The criterion is then implemented as:

$$
\begin{aligned}
& \mathcal{J}\left(x_{1}\right)= \\
& \sum_{k} \frac{1}{4 \sigma_{1}^{2}(k)}\left|I_{1}(k)-\mathcal{F}^{-1}\left[\widetilde{I}_{0}(u) \times \Pi_{u_{\mathrm{c}}}(u) \times \mathrm{e}^{-2 \mathrm{i} \pi u x_{1}}\right]\right|^{2}
\end{aligned}
$$


where $\mathcal{F}^{-1}$ denotes the inverse FFT, $\widetilde{I}$ denotes the FT of an image, $x_{1}$ a real number and $\Pi_{u_{\mathrm{c}}}(u)$ is the low-pass mask in the Fourier domain.

We consider an object that we convolve by a perfect PSF (i.e. the PSF of a perfect telescope without aberrations of turbulence). We then build series of 100 identical images randomly shifted, with a uniform distribution of shift parameters, in each direction. The maximum shift value is 1 since we want to test the sub-pixel accuracy. Moreover, the integer part of the shift parameters can usually be retrieved from the files headers when the shift is induced by a jitter of the instrument. These $100 \mathrm{im}$ ages can then be corrupted by noise.

\subsubsection{Pure stationary Gaussian noise}

In this case we consider only detector noise: Gaussian stationary noise is added to each image with a constant variance of 100 , which is typical of current $\mathrm{AO}$ equipped cameras (e.g. NAOS/CONICA). We then build 5 series of 100 images, increasing the maximum number of photons per pixel in each series, i.e. increasing the $S N R$. Each series is then registered with the three methods (astro-lib, CC and pairwise ML). The reference is taken as one image in each series, so we are in the realistic case of an unknown reference.

The results presented in Fig. 2 bottom demonstrate that, more than being intrinsically sub-pixel, our method exhibits an increase in accuracy with $S N R$ in the images. The performance of the interpolated cross-correlation obviously depends on the interpolation method, and the robust one we used saturates close to 0.3 pixel accuracy. We see here that the interpolation method we used, in the sub-pixel regime appears equivalent to oversampling the images by a factor of 3 or more, as usually done (in IDL astro-Lib registration code, for instance). Nevertheless, none of the two interpolation-based $\mathrm{CC}$ method is able to do better than 0.1 pixel whereas the proposed ML method's accuracy is approximatively inversely proportional to the maximum number of photons per pixel.

We have hence demonstrated the efficiency of our sub-pixel method to register very noisy frames, in the classical case of stationary white Gaussian noise, and considering a noisy image as a reference. We reach the pixel accuracy as the maximum number of photon per pixel is comparable to the variance of the Gaussian noise and can go down to the thousandth of pixel at very high photon levels $\left(10^{5}\right)$, which completely outperforms the classical cross-correlation method and its refinements.

\subsubsection{Mixture of Gaussian and Poissonian noise}

The method has also been tested with a more realistic noise model. The same shifted series of a reference object convolved by a PSF is first corrupted by Poissonian noise. We then add Gaussian stationary noise with a constant variance of 100 . The total variance of the noise is then the shifted reference image (object convolved by the PSF and shifted) plus a constant for the Gaussian noise. We tested the method on 5 series of images with a maximum number of photons per pixel ranging from 10 to $10^{5}$. We present the results in Fig. 3, in the case of known (i.e. a non noisy image of the sequence) and unknown reference (i.e. a noisy image of the sequence) of the ML method. Two methods are tested in each case, one considering a noise variance map, and one considering a constant variance. The aim of this more realistic modeling of the noise is, firstly, to validate the method in the case of a more sophisticated noise model, and secondly to prove the efficiency of the exact knowledge of the noise distribution. We see that in both cases (known and unknown reference), introducing a map of the noise variance slightly increases the accuracy as the max number of photon per pixel $\left(N_{\text {phot }}\right)$ reaches a reasonable value. Indeed, until the max number of photon per pixel is significantly greater than the variance of the detector noise, the total noise variance can be considered as constant, and the accuracy of the two methods is the same. Moreover, in the realistic case of an unknown reference, the advantage of using a true variance is lessened. Finally, we note that the accuracy depends on the $S N R$. Two regimes appear: at low photon level (detector dominated noise, $S N R \propto N_{\text {phot }}$ ), the accuracy is proportional to the inverse of $N_{\text {phot }}$; at high photon level (photon limited noise, $S N R \propto N_{\text {phot }}^{1 / 2}$ the accuracy is proportional to the inverse of the square of $N_{\text {phot }}$. As expected, the transition between the two regimes is smoother in the academic case of the known reference.

\section{Sequences of images: the Joint ML approach}

In this section, we address the problem of registering several (more than 2) images. Actually, it is very rare to have only a couple of noisy images of an object, and the general case is to have a sequence of many images. Intuitively, one should so be able to obtain a better estimation of the shift parameters between images if considering all the sequence. This approach is similar to what was developed by Guillaume et al. (1998) in the case of pure photon noise. We propose here a joint version of our ML registration, in order to estimate simultaneously the non noisy reference image and the shift parameters between the images.

\subsection{Joint estimation of the reference and the shift parameters}

We consider a series of images $\left\{I_{j}(k)\right\}$ randomly shifted. If we try to find simultaneously the shift parameters $\left\{x_{j}\right\}$ and the reference image $R(x)$, then the joint likelihood can be written as:

$$
\begin{aligned}
& \mathcal{L}\left(\left\{I_{j}(k)\right\} ; R(x),\left\{x_{j}\right\}\right) \propto \\
& \prod_{m} \prod_{k} \exp \left(-\frac{1}{2 \sigma_{m}^{2}(k)}\left|I_{m}(k)-\left[R * \delta\left(x-x_{m}\right)\right]_{\amalg}(k)\right|^{2}\right) .
\end{aligned}
$$

And, the neg-log-likelihood to be minimized is then:

$$
\begin{aligned}
& \mathcal{J}\left(\left\{I_{j}(k)\right\} ; R(x),\left\{\mu_{j}\right\}\right)= \\
& \sum_{m} \sum_{k} \frac{1}{2 \sigma_{m}^{2}(k)}\left|I_{m}(k)-\left[R * \delta\left(x-x_{m}\right)\right]_{\amalg}(k)\right|^{2}
\end{aligned}
$$




\subsection{The joint $M L$ estimate of the reference image}

Following the approach of Guillaume et al. (1998), canceling this joint criterion with respect to the reference gives an analytical expression of a reference estimate that minimizes the criterion for a given set of $\left\{x_{j}\right\}$ (see Appendix A):

$\hat{R}_{\mathrm{ML}}\left(k,\left\{\left(x_{m}\right)\right\}\right)=\frac{1}{N_{\text {images }}} \sum_{m}\left[I_{m}(x) * \delta\left(x+x_{m}\right)\right]_{\mathrm{WI}}(k)$.

This is a quite intuitive result, as it is nothing but the average of the shifted-back images. This result is the same as the one obtained by Guillaume et al. (1998) in the case of photon limited data. The criterion obtained by substituting $R$ by its ML estimation $\hat{R}_{\mathrm{ML}}$ depends only on the shifts parameters. Therefore, the criterion to be minimized is given by:

$$
\begin{aligned}
& \mathcal{J}\left(\left\{I_{j}(k)\right\} ; \hat{R}_{\mathrm{ML}}(k),\left\{x_{j}\right\}\right)= \\
& \sum_{m} \sum_{k} \frac{\left|I_{m}(k)-\left[\hat{R}_{\mathrm{ML}}(x) * \delta\left(x-x_{m}\right)\right]_{\amalg}(k)\right|^{2}}{2 \sigma_{m}^{2}(k)} .
\end{aligned}
$$

Additionally, as shown by Blanc et al. (2003), assuming a Gaussian distribution for the object and the noise, we have:

$$
\begin{aligned}
& \mathcal{J}\left(\left\{I_{j}(k)\right\} ; \hat{R}_{\mathrm{ML}}(k),\left\{x_{j}\right\}\right)= \\
& \sum_{m} \sum_{k} \frac{\left|I_{m}(k)-\left[\hat{R}_{\mathrm{ML}}(x) * \delta\left(x-x_{m}\right)\right]_{\amalg}(k)\right|^{2}}{2 \sigma_{m}^{2}(k)}+\mathcal{K}
\end{aligned}
$$

where $\mathcal{K}$ is a corrective term depending on the square modulus of the transfer function $H$ and the object and noise power spectral density. This term is constant in our problem, as we consider that the PSF is the same for all images. The solution of the joint estimator proposed can thus be interpreted as true ML solution of the sole shifts provided the prior distribution of the object is Gaussian.

\subsection{Implementation and validation with simulated images}

The criterion of Eq. (8) is implemented and minimized as in the previous case:

$$
\begin{aligned}
& \mathcal{J}\left(\left\{I_{j}(k)\right\} ; \hat{R}_{\mathrm{ML}}(k),\left\{{ }_{j}\right\}\right)= \\
& \quad \sum_{m} \sum_{k} \frac{1}{2 \sigma_{m}^{2}(k)}\left|I_{m}(k)-\mathcal{F}^{-1}\left[\widetilde{\hat{R}_{\mathrm{ML}}}(u) \times \Pi_{u_{\mathrm{c}}}(u) \mathrm{e}^{-2 \mathrm{i} \pi\left(u x_{1}\right)}\right]\right|^{2}
\end{aligned}
$$

where $\hat{R}_{\mathrm{ML}}$ is given in Eq. (7) and $\Pi_{u_{\mathrm{c}}}(u)$ is the low-pass mask in the Fourier domain cutting at the telescope cutoff frequency $u_{\mathrm{c}}$, the same as the one used in the pairwise ML case. We performed the same kind of simulations as described previously. We compared the pairwise $\mathrm{ML}$ algorithm $(\mathrm{ML} \times 2)$ described in Sect. 2 and the joint estimate with 10 groups of 10 images $(M L \times 10)$ and 1 group of 100 images $(M L \times 100)$. We plotted the average root-mean-square error in each direction for the whole sequence against the max number of photons per pixel in an image. At low photon levels, we see that increasing the number of frames increases the accuracy of the

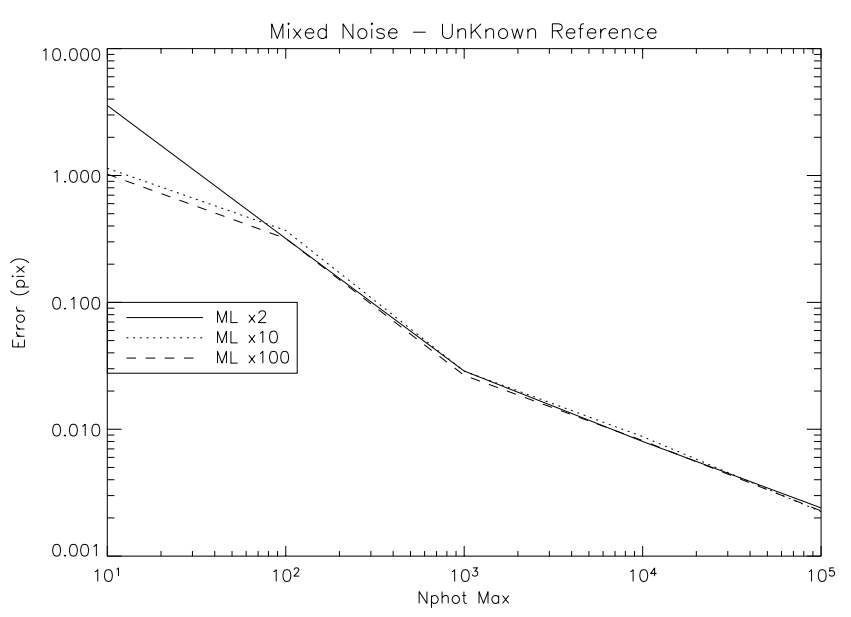

Fig. 4. Performance of the joint estimator compared to the pairwise ML estimator, in the case of mixed (additive Gaussian+Poissonian) noise. The variance of the Gaussian noise is constant: $\sigma_{\text {gauss }}^{2}=$ 100 photons. The number of photons max per pixel ranges from 10 to $10^{5}$.

shift estimation. Actually, we reach a sub-pixel accuracy when the max number of photon per pixel in the image is comparable to the detector noise in the case of a group of 100 images. This has great interest in infrared astronomical imaging where the number of images is usually huge and the $S N R$ is very poor. Note that in the case of a group of 100 images, as expected, the performance are very close to the case of a known reference since the large number of images allows us to retrieve an almost noiseless estimate of the reference.

\section{Application to infrared astronomical images: first images of Arp 220 in the L-band with adaptive optics}

In order to evaluate the performance of our method in a real case, we recorded a sequence of images of a very faint galaxy. Arp 220 is a typical Ultra-Luminous Infrared Galaxy, caracterised by a very powerful emission in the infrared bands but a very faint counterpart at visible wavelengths. Observation of such a galaxy is a challenge as its distance to earth impose high angular resolution imaging in the infrared. NACO (NAOS+CONICA) at the VLT is the only AO system that can servo on infrared sources and thus achieve diffraction limited images of such galaxies in the infrared with a large ground-based telescope (Rousset et al. 2003; Lagrange et al. 2003). A series of 85 images of this galaxy has been acquired in the $L^{\prime}$ band $(3.8 \mu \mathrm{m})$ with this instrument in March 2003. While the background dynamics of each image is around 80000 photons per pixel before sky subtraction, the maximum level on the source is around 100 photons per pixel which is comparable to the total variance of the background after sky subtraction. We are here in the case where the CC was not efficient enough to ensure good registration (accuracy poorer than a pixel). One elementary frame is presented in Fig. 5 as well as the 85 registered and averages frames using the three methods (interpolated cross-correlation, pairwise ML and joint ML. The 


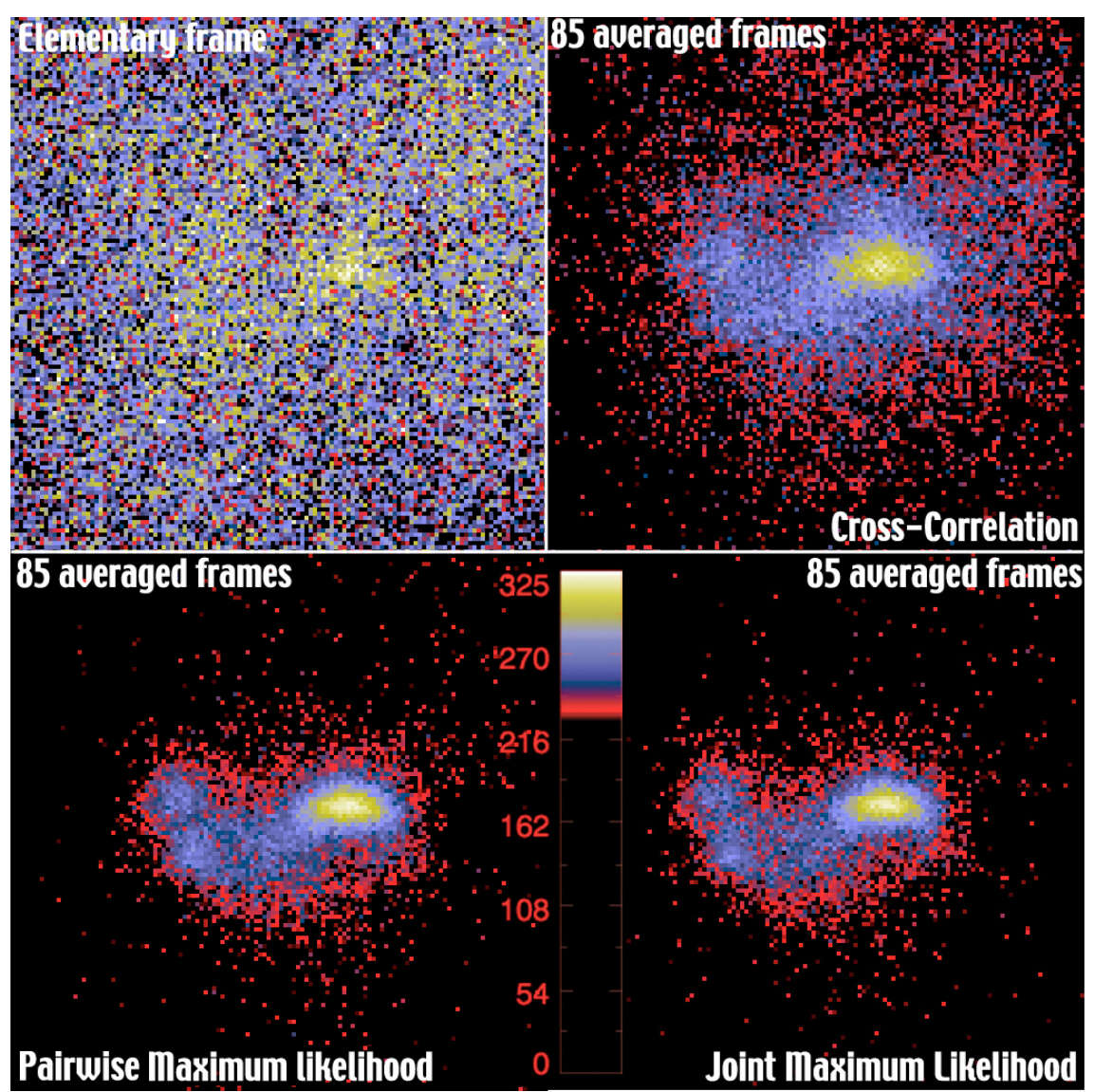

Fig. 5. Adaptive optics image of ARP 220 in the $L$-band with NACO at VLT. Top left: elementary frame, top right: 85 frames registered with a classical cross-correlation method and averaged; bottom left: 85 frames registered with the pairwise ML method and averaged; bottom right: 85 frames registered with the joint ML method and averaged. We chose a log-scale representation to ensure a good contrast for all structures in the image. The color distribution is kept constant for all averaged images to ensure comparison.

obtained Strehl ratio ranged from 0.1 to 0.15 at $2.2 \mu \mathrm{m}$ during the observations meaning 0.25 at $L^{\prime}$.

As obvious in Fig. 5, the images registered with ML methods are better defined than the one obtained with CC. The brightest source intensity is enhanced and it exhibits a clear East-West elongation. Moreover, in the case of the joint ML, it appears slightly bent, similar to the crescent nucleus observed with HST at $2.2 \mu \mathrm{m}$ (Scoville et al. 1998). The surrounding structures show also more details with a clear double-arm pattern linking the two secondary sources to the brightest elongated source which was not obvious on the CC registered image, and globally, the joint ML method gives slightly sharper results than the pairwise method.

The comparison of the circular mean of the Fourier transforms of each image is displayed in the left part of Fig. 6 and shows more quantitatively the improvement obtained with both ML methods. High spatial frequencies are enhanced especially in the range 0.1 to 0.4 (in units of the cutting frequency of the telescope) compared to the $\mathrm{CC}$ method. The improvement obtained with the joint ML compared to the pairwise is also visible just below and around frequency 0.1 .

The previous $L$-band images, obtained without adaptive optics on a 10-m class telescope (Soifer et al. 1999) are similar to the result obtain with $\mathrm{CC}$ registration. The super-imposition of the contour of their study on our image is presented on the right side of Fig. 6 (inset A). In their image, neither the two secondary sources nor the double-arm pattern were clearly defined. Clearly, the details we now obtain with the ML methods at $3.8 \mu \mathrm{m}$ allow a more precise study of the secondary structures and their link to the brightest source. Precise photometry of these sources are now possible and can be compared to the same pattern found by HST at $2.2 \mu \mathrm{m}$ and by Keck $12 \mu \mathrm{m}$ as shown in insets B and C of Fig. 6. Very preliminary morphological interpretations were presented in Gratadour et al. (2003) and a precise photometric study including AO observations at $2.2 \mu \mathrm{m}$ as well as more a refined interpretation will be presented in a future paper in preparation.

\section{Conclusion}

In this paper, we have formulated and tested by means of simulations a ML approach for the registration of shifted images of the same object observed with a single instrument. We have studied the performance of this method as a function of the $S N R$ of the images. We have then demonstrated the efficiency of our ML method to register two noisy images at a sub-pixel accuracy, even when the signal is very low.

In order to obtain a higher accuracy when registering a large amount of very noisy elementary frames, we developed and 

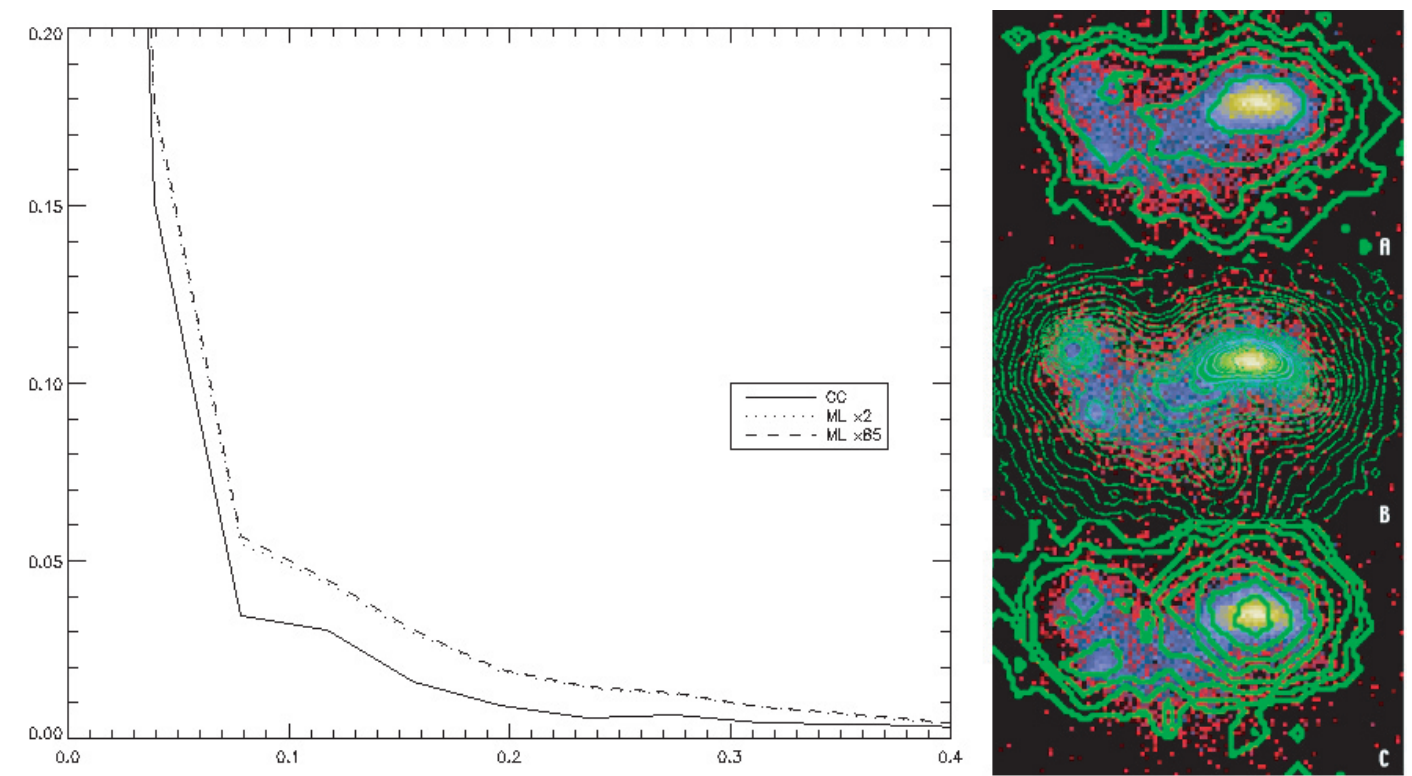

Fig. 6. Left: circular means of the Fourier transforms of the final images obtained with the cross-correlation (CC), the pairwise ML $(\mathrm{ML} \times 2)$ and the joint ML $(\mathrm{ML} \times 85)$ methods. Right: super-imposition of our joint ML image with contours at other wavelengths: A, $3.8 \mu \mathrm{m}$ with Keck, B, $2.2 \mu \mathrm{m}$ with HST and C, $12 \mu \mathrm{m}$ with Keck.

tested a joint ML approach which estimates simultaneously the reference image and the shift parameters between the frames. By deriving analytically the ML solution for the reference image, we obtain a criterion depending on the sole shift parameters which allows a fast and precise minimization. We demonstrated the efficiency of this joint ML estimate at low SNRs, and noted the similarity between this joint ML method and that of the pairwise ML at high SNRs. Finally, we registered experimental astronomical images of a faint galaxy and showed the ability of our ML approach to preserve the resolution obtained in each elementary images thanks to the AO after averaging all the frames.

\section{Appendix A: The ML solution in the case of stationary white Gaussian noise}

In this appendix, we recall how, with a mild assumption (periodicity of the images), in the case of stationary white Gaussian noise, the pairwise ML solution of the registration problem is the maximum of the cross-correlation. Indeed, an analytical expression of the gradient of the negative log-likelihood $\mathcal{J}$ with respect to $x_{1}$, assuming $\sigma_{1}$ is a constant, is given by:

$$
\begin{aligned}
& \nabla_{x_{1}} \mathcal{J}\left(x_{1}\right) \propto \\
& \sum_{k}\left[I_{1}(k)-\left[R\left(x-x_{1}\right)\right]_{\amalg}(k)\right]\left[\frac{\partial}{\partial x_{1}}\left[R\left(x-x_{1}\right)\right]_{\amalg}(k)\right] .
\end{aligned}
$$

Under the assumption that the reference is periodic or not truncated (i.e. an object surrounded by a dark background larger than the largest possible shift): $\sum_{k}\left|[R]_{\amalg}(k)\right|^{2}=$ const. we have:

$$
\sum_{k}\left\{\frac{\partial}{\partial x_{1}}\left[R\left(x-x_{1}\right)\right]_{\amalg}(k)\right\}\left[R\left(x-x_{1}\right)\right]_{\amalg}(k)=0
$$

and, nulling $\nabla_{x_{1}} \mathcal{J}\left(x_{1}\right)$ is then equivalent to solving

$$
\sum_{k}\left\{\frac{\partial}{\partial x_{1}}\left[R\left(x-x_{1}\right)\right]_{\amalg}(k)\right\} I_{1}(k)=0
$$

i.e., to find the maximum of:

$$
C\left(I_{1}, R\right)=\sum_{k} I_{1}(k)\left[R\left(x-x_{1}\right)\right]_{\amalg}(k)
$$

which is nothing but the linear cross-correlation function of the image and the reference.

\section{Appendix B: The ML estimation of the reference in the joint $M L$ case}

In this appendix, we derive the analytical expression of the ML estimate of the reference in the joint ML approach by derivating the joint ML criterion with respect to the reference. We recall the expression of the joint ML criterion:

$$
\begin{aligned}
& \mathcal{J}\left(\left\{I_{j}(k)\right\} ; R(k),\left\{x_{j}\right\}\right)= \\
& \sum_{m} \sum_{k} \frac{\left|I_{m}(k)-\left[R(x) * \delta\left(x-x_{m}\right)\right]_{\amalg}(k)\right|^{2}}{2 \sigma_{m}^{2}(k)} .
\end{aligned}
$$

The maximum likelihood estimate $\hat{R}_{\mathrm{ML}}(x)$ of the reference image, is obtained by nulling $\forall l$ :

$$
\begin{aligned}
& \frac{\partial}{\partial R(l)} \mathcal{J}\left(\left\{I_{j}(k)\right\} ; R(x),\left\{x_{j}\right\}\right)= \\
& \quad \frac{\partial}{\partial R(l)} \sum_{m} \sum_{k} \frac{1}{2 \sigma_{m}^{2}(k)}\left|I_{m}(k)-\left[R * \delta\left(x-x_{m}\right)\right]_{\amalg}(k)\right|^{2}
\end{aligned}
$$


If we consider Nyquist sampled images then we can write: Minimizing

$R(x)=\sum_{n \in \mathbb{Z}} \operatorname{sinc}(x-n) R(n)$

and:

$[R(x)]_{\amalg}(k)=R(x) \times \delta(x-k)$

and:

$\left[R(x) * \delta\left(x-x_{m}\right)\right]_{\amalg}(k)=\sum_{n \in \mathbb{Z}} \operatorname{sinc}\left(k-x_{m}-n\right) R(n)$

so:

$\frac{\partial}{\partial R(l)}\left[R(x) * \delta\left(x-x_{m}\right)\right]_{\mathrm{W}}(k)=\operatorname{sinc}\left(k-x_{m}-l\right)$.

Then, we have:

$\frac{\partial}{\partial R(l)} \mathcal{J}\left(\left\{I_{j}(k)\right\} ; R(x),\left\{x_{j}\right\}\right)=$

$\sum_{m} \sum_{k} \frac{-1}{\sigma_{m}^{2}(k)} \operatorname{sinc}\left(k-x_{m}-l\right)\left[I_{m}(k)-\left[R(x) * \delta\left(x-x_{m}\right)\right]_{\amalg}(k)\right]$.

We see that:

$\sum_{k} \frac{1}{\sigma_{m}^{2}(k)} \operatorname{sinc}\left(k-x_{m}-l\right) I_{m}(k)=$

$$
\sum_{k} \frac{1}{\sigma_{m}^{2}(k)} \operatorname{sinc}\left(l+x_{m}-k\right) I_{m}(k)=\left[\frac{I_{m}\left(x+x_{m}\right)}{\sigma_{m}^{2}\left(x+x_{m}\right)}\right]_{\amalg}(l)
$$

and, assuming that $R$ is Nyquist sampled,

$$
\sum_{k} \frac{\left[R\left(x-x_{m}\right)\right]_{\amalg}(k)}{\sigma_{m}^{2}(k)} \operatorname{sinc}\left(k-x_{m}-l\right)=\left[\frac{R(x)}{\sigma_{m}^{2}\left(x+x_{m}\right)}\right]_{\amalg}(l) .
$$

So we see that $\forall l$ :

$$
\begin{aligned}
\frac{\partial}{\partial R(l)} \mathcal{J}\left(\left\{I_{j}(k)\right\} ; R(x),\left\{x_{j}\right\}\right) & =0 \Leftrightarrow \\
\sum_{m}\left[\frac{I_{m}\left(x+x_{m}\right)}{\sigma_{m}^{2}\left(x+x_{m}\right)}\right]_{\amalg}(l) & =\sum_{m}\left[\frac{R(x)}{\sigma_{m}^{2}\left(x+x_{m}\right)}\right]_{\amalg}(l) .
\end{aligned}
$$

As argued in Sect. 2.2.3 $\sigma_{m}^{2}(x) * \delta\left(x-x_{m}\right)=\sigma_{n}^{2}(x) * \delta\left(x-x_{n}\right)$. Then, we can write:

$\sum_{m}\left[I_{m}\left(x+x_{m}\right)\right]_{\amalg}=\sum_{m}[R(x)]_{\amalg}$

and so:

$\hat{R}_{\mathrm{ML}}(k)=\frac{1}{N_{\text {images }}} \sum_{m}\left[I_{m}(x) * \delta\left(x+x_{m}\right)\right]_{\mathrm{W}}(k)$.
$\mathcal{J}\left(\left\{I_{j}(k)\right\} ; R(x),\left\{x_{j}\right\}\right)$

on $R(x)$ and $\left\{x_{j}\right\}$ is equivalent to minimize:

$\mathcal{J}\left(\left\{I_{j}(k)\right\}, R(k)=\hat{R}_{\mathrm{ML}}(k) ;\left\{x_{j}\right\}\right)$

on $\left\{x_{j}\right\}$ only as discussed in Sect. 3.2.

\section{References}

Blanc, A., Mugnier, L. M., \& Idier, J. 2003, J. Opt. Soc. Am. A, 20, 1035

Brown, L. G. 1992, ACM Compting Surveys, 4, 325

Carfantan, H., \& Rougé, B. 2001, in XVIII ${ }^{\mathrm{e}}$ GRETSI, 849

Costa, W. L. S., Haynor, D. R., Haralick, R. M., Lwellen, T. K., \& Graham, M. M. 1993, in IEEE Nuclear Science Symp. and Medical Imaging Conf., 974, 38

Fruchter, A. S., \& Hook, R. N. 2002, PASP, 114, 144

Girémus, A., \& Carfantan, H. 2003, in XIX ${ }^{\mathrm{e}}$ GRETSI, 2

Gratadour, D., Rouan, D., Clenet, Y., Gendron, E., \& Lacombe, F. 2003, in IAU Symp., 221

Guillaume, M., Melon, P., Refregier, P., \& Llebaria, A. 1998, J. Opt. Soc. Am. A, 15, 2841

Lagrange, A., Chauvin, G., Fusco, T., et al. 2003, in Instrument Design and Performance for Optical/Infrared Ground-based Telescopes, ed. M. Iye, \& A. F. M. Moorwood, Proc. SPIE, 4841, 860

Maintz, J. B. A., \& Viergever, M. A. 1998, Medical Image Analysis, 2,1

Maurer, C. R., \& Fitzpatrick, J. M. 1993, Interactive imageguided neurosurgery, ed. R. J. Maciunas, American Association of Neurological Surgeons, Park Ridge, IL, 17

Mort, M. S., \& Srinath, M. D. 1988, in Applications of digital image processing, IEEE Proc. of SPIE, 974, 38

Mugnier, L. Z., Fusco, T., \& Conan, J. 2004, JOSAA, in press

Roche, A., Malandain, G., \& Ayache, N. 2000, Int. J. Imaging Syst. Techn., 11, 71

Rousset, G., Lacombe, F., Puget, P., et al. 2003, in Adaptive Optical System Technologies II, ed. P. L. Wizinowich, \& D. Bonaccini, Proc. SPIE, 4839, 140

Scoville, N. Z., Evans, A. S., Dinshaw, N., et al. 1998, ApJ, 492, L107

Snyder, D. L., Helstrom, C. W., Lanterman, A. D., Faisal, M., \& White, R. L. 1995, OSAJ, 12, 272

Snyder, D. L., \& Schulz, T. 1990, OSAJ, 7, 1251

Soifer, B. T., Neugebauer, G., Matthews, K., et al. 1999, ApJ, 513, 207

Van Trees, H. L. 1968, Detection, Estimation and Modulation theory Part I (John Wiley \& Sons)

Zitová, B., \& Flusser, A. 2003, Image and Vision Computing, 21, 977 